Network Working Group

Request for Comments: 3085

Category: Informational
A. Coates

Reuters

D. Allen

IPTC

D. Rivers-Moore

Rivcom

March 2001

\title{
URN Namespace for NewsML Resources
}

Status of this Memo

This memo provides information for the Internet community. It does not specify an Internet standard of any kind. Distribution of this memo is unlimited.

Copyright Notice

Copyright (C) The Internet Society (2001). All Rights Reserved.

Abstract

This document describes a URN (Uniform Resource Name) namespace for identifying NewsML NewsItems. A NewsItem is an information resource that is expressible as a NewsML element within a NewsML document conforming to the NewsML Document Type Declaration (DTD) as defined by the International Press Telecommunications Council (IPTC) .

1. Introduction

NewsML is an XML format for packaging multimedia news resources. It has been created under the auspices of the International Press Telecommunications Council (IPTC), and version 1.0 was approved by the IPTC on 6 october 2000 .

The same logical NewsItem may exist in multiple physical locations. The NewsML specification allows NewsItems to have multiple URLs, but only a single URN. It is the latter which then uniquely names the resource.

This namespace specification is for a formal namespace.

2. Specification Template

Namespace ID:

"newsml" requested. 
Registration Information:

Registration Version Number: 1

Registration Date: 2000-11-07

Declared registrant of the namespace:

David Allen

ho73edial.pipex.com

IPTC

Royal Albert House

Sheet Street

Windsor

SL4 1BE

Declaration of structure:

The identifier has the following ABNF[6] specification:

NSS = ProviderId ":" DateId ":" NewsItemId ":" RevisionId Update ProviderId = string

DateId = date

NewsItemId $=$ string

RevisionId = posint

Update $=0 * 1$ ( "A" / "U" )

date $=$ century year month day

century $=$ ( "0" posdig ) / ( posdig DIGIT )

year $=1 * 2 \mathrm{DIGIT}$

month $=(0$ posdig $) /(" 1 "$ ( "0" "1" "2" ) )

day $=(0$ posdig $) /(" 1 " / 4 "$ / DIGIT ) / "30" / "31"

string $=1{ }^{*}$ char

char = ALPHA / DIGIT / symbol / escape

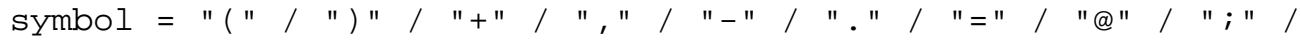

"\$" / " " / " " / " " / " "

escape $=\overline{\text { "응 }}$ HEXDIG HEXDIG

posint $=$ posdig $\star$ DIGIT

posdig ="1" / "2" / "3" / "4" / " / / " / " / " / " " " "

The ProviderId must be an Internet domain name, and must be owned by the organisation creating the NewsML resource and allocating the URN to it, at the date identified by the DateId.

DateId is a date in ISO 8601 Basic Format (CCYYMMDD), and must correspond to a date at which the organisation allocating the URN owned the domain name specified in the ProviderId.

The NewsItemId must be unique among all NewsItems emanating from the same provider and having the same DateId. 
RevisionId is a positive integer indicating which revision of a given NewsItem this is. Any positive integer may be used, but it must always be the case that of two instances of a NewsItem that have the same ProviderId, DateId and NewsItemId, the one whose RevisionId has the larger value must be the more recent revision. A RevisionId of 0 is not permitted.

If the Newsitem contains an "Update" element or elements, then Update must be set to "U". If the NewsItem consists only of a replacement set of NewsManagement data, then Update must be set to "A". If neither of these is the case, then Update must be suppressed.

Relevant ancillary documentation:

None

Identifier uniqueness considerations:

The combination of ProviderId and Dateld serves to uniquely identify the organisation that is allocating the URN. That organisation is responsible for ensuring the uniqueness of the DateId/NewsItemId/RevisionId combination.

Identifier persistence considerations:

A NewsML URN may only be allocated by an organisation that owns an Internet domain name. The URN identifies a date on which the organisation owned that domain name. The combination of date and domain name will serve to uniquely identify that organisation for all time.

Process of identifier assignment:

The organisation identified by the ProviderId/DateId combination is responsible for allocating a NewsitemId that is unique among all those that it allocates with that DateId.

Process of identifier resolution:

NewsML providers are responsible for the provision of a URN resolution service, if any, for NewsML URNs they have assigned with a valid ProviderId/DateId combination.

Rules for Lexical Equivalence:

URNs are lexically equivalent if the ProviderId, DateId, NewsItemId, and RevisionId are all identical (case-insensitive comparison). 
Conformance with URN Syntax:

No special considerations beyond the syntax herein described.

Validation mechanism:

Organisations that allocate NewsML URNs are responsible for the provision of a URN validation service, if any, for URNs they have assigned with a valid ProviderId/DateId combination.

Scope :

Global

3. Examples

The following examples are representative of NewsML URNs, but may not refer to actual resources.

urn: newsml:iptc.org:20001006:NewsMLv1.0:1

urn:newsml:reuters.com:20000206:

$$
\text { IIMFFH05643_2000-02-06_17-54-01_L06156584:1U }
$$

4. Security Considerations

There are no additional security considerations other than those normally associated with the use and resolution of URNs in general.

References

[1] Rivers-Moore, D., "NewsML Version 1.0 Functional Specification", November 2000,

<http://www.iptc.org/NewsML/specification/NewsMLv1.0.pdf>.

[2] Rivers-Moore, D., "NewsML Version 1.0 DTD", November 2000, <http://www.iptc.org/NewsML/DTD/NewsMLv1.0.dtd>.

[3] W3C, XML WG, "Extensible Markup Language (XML) 1.0", February 1998, <http://wWw.w3.org/TR/REC-xml>.

[4] Moats, R., "URN Syntax", RFC 2141, May 1997.

[5] Daigle, L., van Gulik, D., Iannella, R. and P. Faltstrom, "URN Namespace Definition Mechanisms", RFC 2611, June 1999.

[6] Crocker, D. and P. Overell, "Augmented BNF for Syntax Specifications: ABNF", RFC 2234, November 1997. 


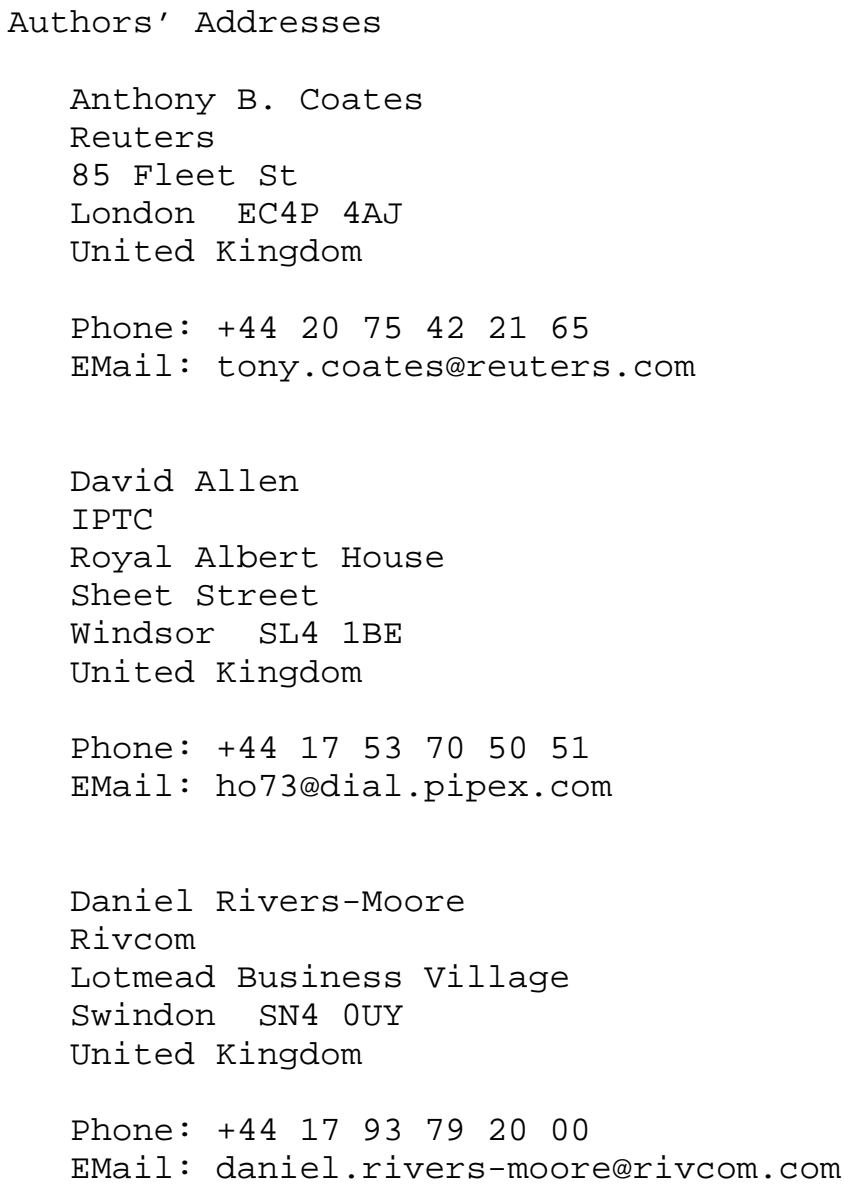


Full Copyright statement

Copyright (C) The Internet Society (2001). All Rights Reserved.

This document and translations of it may be copied and furnished to others, and derivative works that comment on or otherwise explain it or assist in its implementation may be prepared, copied, published and distributed, in whole or in part, without restriction of any kind, provided that the above copyright notice and this paragraph are included on all such copies and derivative works. However, this document itself may not be modified in any way, such as by removing the copyright notice or references to the Internet society or other Internet organizations, except as needed for the purpose of developing Internet standards in which case the procedures for copyrights defined in the Internet Standards process must be followed, or as required to translate it into languages other than English.

The limited permissions granted above are perpetual and will not be revoked by the Internet society or its successors or assigns.

This document and the information contained herein is provided on an "AS IS" basis and THE INTERNET SOCIETY AND THE INTERNET ENGINEERING TASK FORCE DISCLAIMS ALL WARRANTIES, EXPRESS OR IMPLIED, INCLUDING BUT NOT LIMITED TO ANY WARRANTY THAT THE USE OF THE INFORMATION HEREIN WILL NOT INFRINGE ANY RIGHTS OR ANY IMPLIED WARRANTIES OF MERCHANTABILITY OR FITNESS FOR A PARTICULAR PURPOSE.

Acknowledgement

Funding for the RFC Editor function is currently provided by the Internet society. 